\title{
First-in-Human Use of a Hybrid Real-Time Ultrasound-Guided Fine-Needle Acquisition System for Peripheral Pulmonary Lesions: A Multicenter Pilot Study
}

\author{
Lonny B. Yarmus ${ }^{a}$ Christopher Mallow ${ }^{a}$ Nicholas Pastis $^{b}$ Jeffrey Thiboutot $^{a}$ \\ Hans Lee ${ }^{a}$ David Feller-Kopman ${ }^{a}$ Andrew D. Lerner ${ }^{a}$ Nicole Tanner ${ }^{b}$ \\ Gerard Silvestri $^{\mathrm{b}}$ Alexander Chen ${ }^{c}$ on behalf of the Interventional Pulmonary \\ Outcomes Group (IPOG)

\begin{abstract}
a Section of Interventional Pulmonology, Division of Pulmonary and Critical Care Medicine, Johns Hopkins University School of Medicine, Baltimore, MD, USA; ${ }^{\mathrm{b}}$ Division of Pulmonary and Critical Care Medicine, Medical University of South Carolina, Charleston, SC, USA; 'Section of Interventional Pulmonology, Division of Pulmonary and Critical Care Medicine, Washington University School of Medicine, St. Louis, MO, USA
\end{abstract}

\section{Keywords}

Peripheral pulmonary lesions · Bronchoscopy ·

Ultrasound-guided fine-needle aspiration

\begin{abstract}
Background: The ability to successfully perform a biopsy on pulmonary lesions by means of bronchoscopy varies widely due to anatomic and technological limitations. One major limitation is the lack of the ability to utilize real-time guidance during tissue sampling in the periphery. A novel system has been developed that enables real-time visualization and sampling of peripheral lesions by displaying an ultrasound image of the lesion and needle simultaneously. Methods: We performed a multicenter, prospective pilot in patients with peripheral pulmonary lesions undergoing a clinically indicated bronchoscopy. The purpose of this study was to demonstrate the feasibility of visualizing, accessing, and obtaining specimens adequate for the cytology of lung lesions when using a novel hybrid real-time ultrasound-guided fine-
\end{abstract}

\section{KARGER}

(c) 2019 S. Karger AG, Basel

E-Mail karger@karger.com

www.karger.com/res needle aspiration system for peripheral pulmonary lesions. Results: Twenty-three patients underwent bronchoscopic sampling of a peripheral pulmonary lesion with the study device. Mean lesion size was 3.6 (range 1.7-5.7) cm. Targeted lesions were located in all lobes of the lung. All lesions were successfully visualized and sampled under real-time visualization with specimens adequate for cytological evaluation. The needle was visualized in all lesions throughout targeting and sampling. There were no incidents of pneumothorax or moderate-to-severe bleeding. Conclusion: In this feasibility study, we report the first-in-human use of a continuous realtime endobronchial ultrasound guidance system to sample peripheral pulmonary lesions. Future generations of this device may improve usability and further studies are needed to determine the true diagnostic capabilities of this novel technique.

(c) 2019 S. Karger AG, Basel

Drs. Yarmus and Mallow contributed equally to this manuscript as first authors. The trial was registered on ClinicalTrials.gov (NCT02832284).
Lonny Yarmus, DO, MBA

Associate Professor of Medicine and Oncology

Johns Hopkins University School of Medicine, 1830 E. Monument Street

Baltimore, MD 21205 (USA)

E-Mail lyarmus@jhmi.edu 


\section{Introduction}

The most common techniques performed for the diagnosis of peripheral lung lesions are bronchoscopy, surgical biopsy, and computed tomography-guided transthoracic-needle biopsy (CT-TTNA) [1]. Though surgical and CT-guided biopsies are often conclusive, they can lead to significant morbidity $[2,3]$. Surgical biopsy has the highest diagnostic yield but also suffers from higher morbidity than CT-guided biopsy or bronchoscopy [24]. Although less invasive than surgery, CT-TTNA carries a higher risk of pneumothorax than bronchoscopy [5].

Less invasive techniques for obtaining diagnoses for peripheral pulmonary lesions are performed primarily by using bronchoscopy with conventional radial endobronchial ultrasound (CR-EBUS) or electromagnetic navigational bronchoscopy (ENB). CR-EBUS requires the use of an ultrasound catheter, which is positioned until visualization of the lesion is confirmed. The catheter is subsequently removed, and the sampling instruments are then advanced blindly without ultrasound/image guidance into the distal airways to obtain diagnostic tissue. This procedure is safer than surgery or CT-TTNA but has a lower diagnostic yield that ranges from 46 to $86 \%$ [6-9]. The lower diagnostic yield of guided bronchoscopy may be due to multiple factors including the absence of real-time imaging during sample acquisition and respiratory variation along with nodule target movement [10].

ENB uses virtual bronchoscopy, in combination with an electromagnetic field, to provide guidance during bronchoscopic sampling. Using a preprocedural CT scan and a software-based tracking system, the bronchoscopist is able to navigate to the peripheral nodule using computer-generated airway mapping. The diagnostic yield of $\mathrm{ENB}$ in the literature is highly variable, ranging from 38.5 to $94 \%[8,11,12]$. Similar to with CR-EBUS, there is also no real-time visualization of lesions since the image guidance and tracking are computer-generated and based on preprocedural CT mapping.

While central nodules are easier to biopsy in real-time using convex EBUS-guided transbronchial-needle aspiration (TBNA), we currently lack the technology to obtain tissue of peripheral lesions under direct visualization. We evaluated the safety and early efficacy of a novel radial EBUS prototype device with a needle located adjacent to the catheter (Fig. 1), which allows for real-time positioning of the needle and tissue acquisition from peripheral nodules using TBNA (RT-EBUS-TBNA) (Boston Scientific, Marlborough, MA, USA).

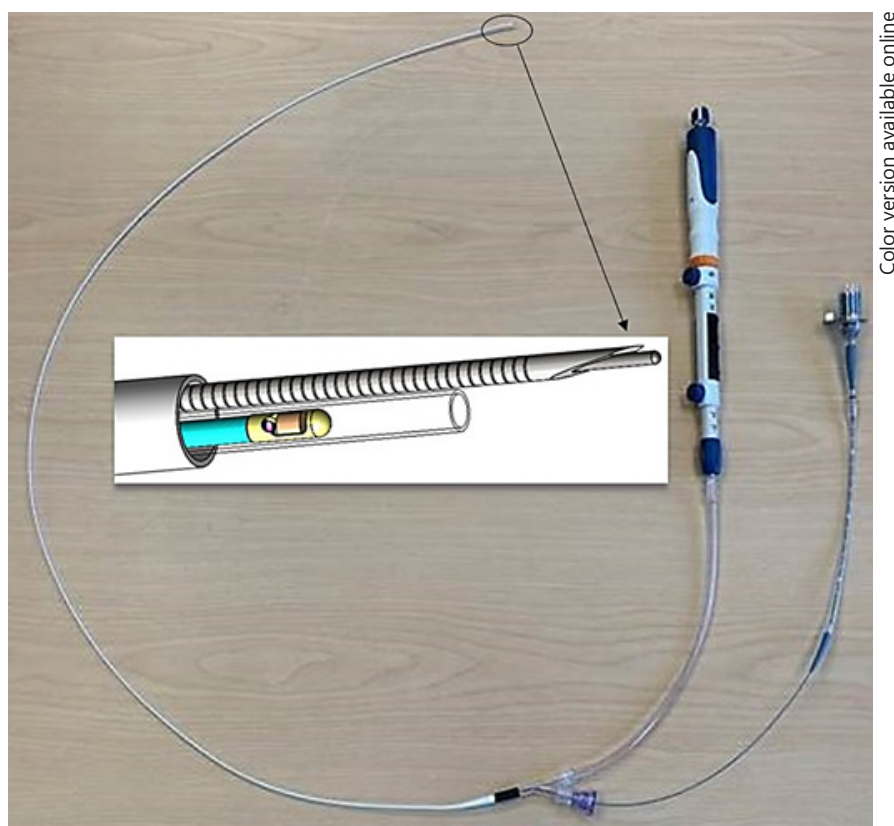

Fig. 1. Real-time radial endobronchial ultrasound biopsy tool. The magnified insert illustration shows the proximity of the needle to the ultrasound that allows for real-time visualization of lesion sampling.

\section{Methods}

This was a multicenter, prospective, safety and feasibility pilot study conducted by the Johns Hopkins University (Baltimore, $\mathrm{MD}$ ), the Washington University School of Medicine (St. Louis, $\mathrm{MO}$ ), and the Medical University of South Carolina (Charleston, SC), USA, on the use of a novel radial EBUS device with a real-time sampling needle for the biopsy of peripheral pulmonary nodules from 18 January to 8 June 2017.

Inclusion criteria included an age of $>18$ years, a predominantly solid lung lesion (at least $80 \%$, i.e., $1-7 \mathrm{~cm}$ in diameter) identified on chest CT, the intention to undergo bronchoscopy evaluation under routine clinical care, and the decision to pursue biopsy. Participants were excluded if the lesion was $>20 \%$ ground-glass or sub-solid, or if there was endobronchial involvement on chest CT, and if the patient was unable to undergo bronchoscopy, had a known coagulopathy, was pregnant or a nursing mother, or was currently enrolled in another investigational study.

At the baseline visit, informed consent, medical history, and a chest CT image obtained within 6 weeks of the planned study procedure to confirm the presence of peripheral pulmonary lesions were recorded. All patients initially underwent convex EBUS-TBNA for mediastinal and hilar staging. This was followed by the study procedure where the participant had tissue sampling using the novel RT-EBUS-TBNA system under deep sedation.

The RT-EBUS-TBNA system includes a specially constructed, flexible sheath with a radial ultrasound catheter as well as a specially engineered needle to be passed through the same sheath to establish real-time visualization of lesion sampling (Boston Scientific). The device has an outer diameter of $1.9 \mathrm{~mm}$ so as to be com- 


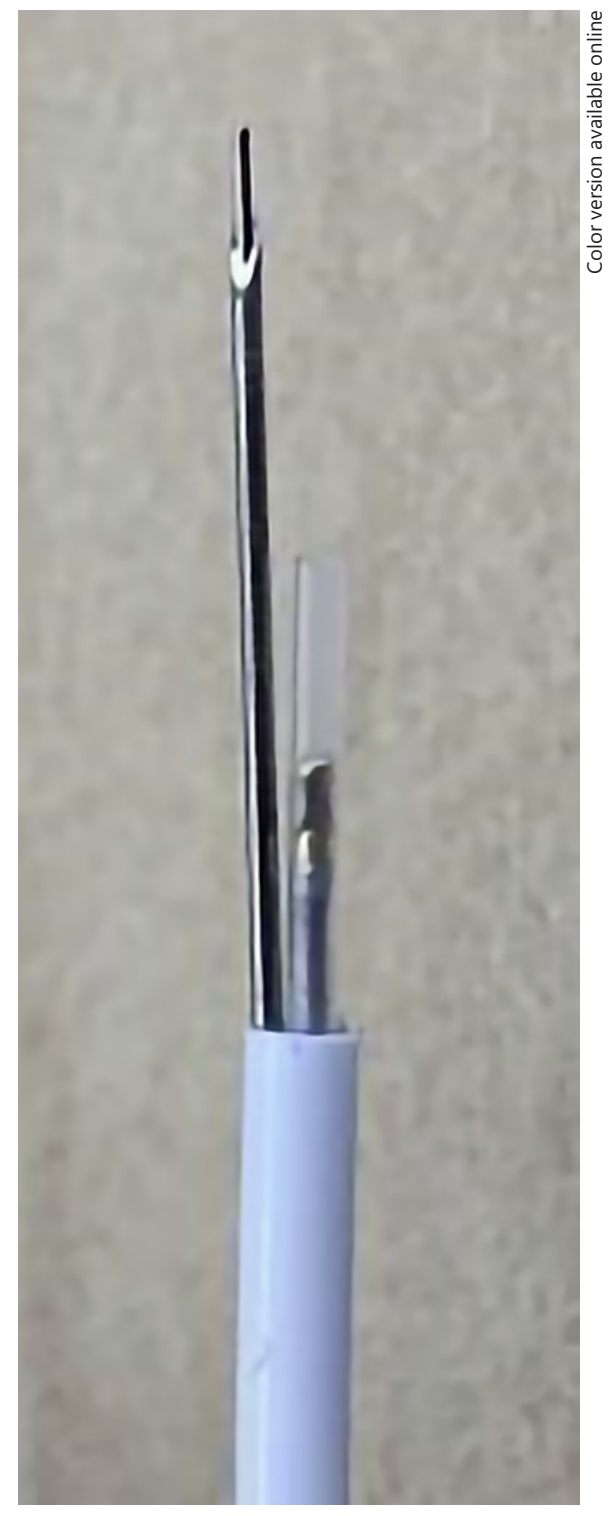

Fig. 2. Distal tip of radial endobronchial ultrasound biopsy tool with a $10^{\circ}$ separation between the ultrasound probe and the needle.

patible with a $2.0-\mathrm{mm}$ working channel. The sheath is comprised of a disposable $40-\mathrm{MHz}, 1.1-\mathrm{mm}$ ultrasound probe with a 25-gauge needle in a single lumen (Boston Scientific).

All participants underwent flexible bronchoscopy with the investigational RT-EBUS-TBNA biopsy system under fluoroscopic guidance using a $4.2-\mathrm{mm}$ bronchoscope with a $2.0-\mathrm{mm}$ working channel (Olympus BF-P190 or MP-160). Nodule localization was accomplished using CT scan anatomic guidance and fluoroscopy. No virtual or electromagnetic navigation platforms were used, although since the outer diameter of the device was $1.9 \mathrm{~mm}$ it could, conceptually, be used with any approximately sized extended working channel $>2.0 \mathrm{~mm}$. Once the bronchoscope was positioned adjacent to the target, the guide sheath which included the RT-EBUSTBNA device was passed through the working channel of the bron- choscope, and the radial ultrasound probe was then advanced into the parenchyma using the same technique as CR-EBUS under fluoroscopic guidance [6]. No curette device was used. Once an ultrasound view was obtained, the probe was manipulated to obtain the optimal image for nodule access. Under fluoroscopic guidance, the TBNA needle was then deployed under direct ultrasound visualization, revealing the needle position as either concentric or eccentric to the lesion; this was feasible due to the design of the device which allowed for separation of the radial probe and needle at approximately $10^{\circ}$ (Fig. 2). If the needle was concentrically placed, cytologic samples were obtained as described below. If the needle was eccentric to the lesion, the needle was retracted and the entire sheathed device allowed for the rotation and repositioning of the device, at which point the needle was redeployed in the correct plane and samples were obtained. The needle possesses a stroke limiter on the handle to limit its depth to a maximum of $2.5 \mathrm{~cm}$ to limit the risk of overextension and potential pleural injury.

All samples obtained were assessed using rapid on-site evaluation (ROSE) for specimen adequacy as well as diagnostic yield. After a RT-EBUS-TBNA sample was taken, the aspirate was expressed onto glass slides. The first smear was air-dried and stained immediately with a rapid Giemsa-type stain (Diff-Quick [DQ], Fisher Scientific, Kalamazoo, MI, USA) and the second was fixed in 95\% alcohol for permanent cytologic examination using the Papanicolaou stain. Criteria for ROSE sample adequacy was defined as the presence of cellular material which could be stained and evaluated by the onsite cytologist and/or a diagnostic sample (e.g., adenocarcinoma). If the sample contained no cellular material (i.e., blood only), it was considered inadequate on ROSE. Diagnostic yield was defined as the fraction of lesion(s) for which a diagnosis could be obtained based on a multidisciplinary evaluation of all information available, including but not limited to the analysis of the RT-EBUS-TBNA aspirate. A sample was considered diagnostic during the index procedure if ROSE evaluation demonstrated a specific malignant or benign diagnosis (e.g., granulomatous inflammation consistent with sarcoidosis). The final pathology reports were considered the diagnostic gold standard. If ROSE samples were inadequate for cytological evaluation or the samples were not diagnostic on-site as defined above, additional standard of care procedures were performed using CR-EBUS with TBNA, brush, and/or transbronchial biopsy.

The primary end point was the ability to acquire adequate specimens of cellular material suitable for cytological evaluation of the targeted lesion under real-time visualization as defined above. Secondary end points included the occurrence and severity of adverse events (AEs) related to the procedure, AEs related to any subsequent standard of care technique, the proportion of lesions visualized using the RT-EBUS-TBNA system, the proportion of lesions accessed where RT-EBUS biopsy needles were deployed during the study maneuvers, and the proportion of RT-EBUS maneuvers that acquired specimens of cellular matter for cytology.

Descriptive statistics were used to summarize the end points of this prospective observational pilot study. Mean (with standard deviation) was used to describe continuous variables with a normal distribution, and median (with interquartile range) was used to describe continuous variables with a skewed distribution. Frequency tables were used to summarize discrete variables, and proportions were used for AE data. We conducted statistics using an intentionto-treat (ITT) cohort, consisting of subjects enrolled in the study regardless of whether a specimen was obtained by RT-EBUS. Statistical analysis was conducted using SAS v9.3 (Cary, NC, USA). 
Fig. 3. a Fluoroscopic view of RT-EBUSTBNA adjacent to a pulmonary lesion. b RT-EBUS view with the TBNA needle visualized entering the lesion.
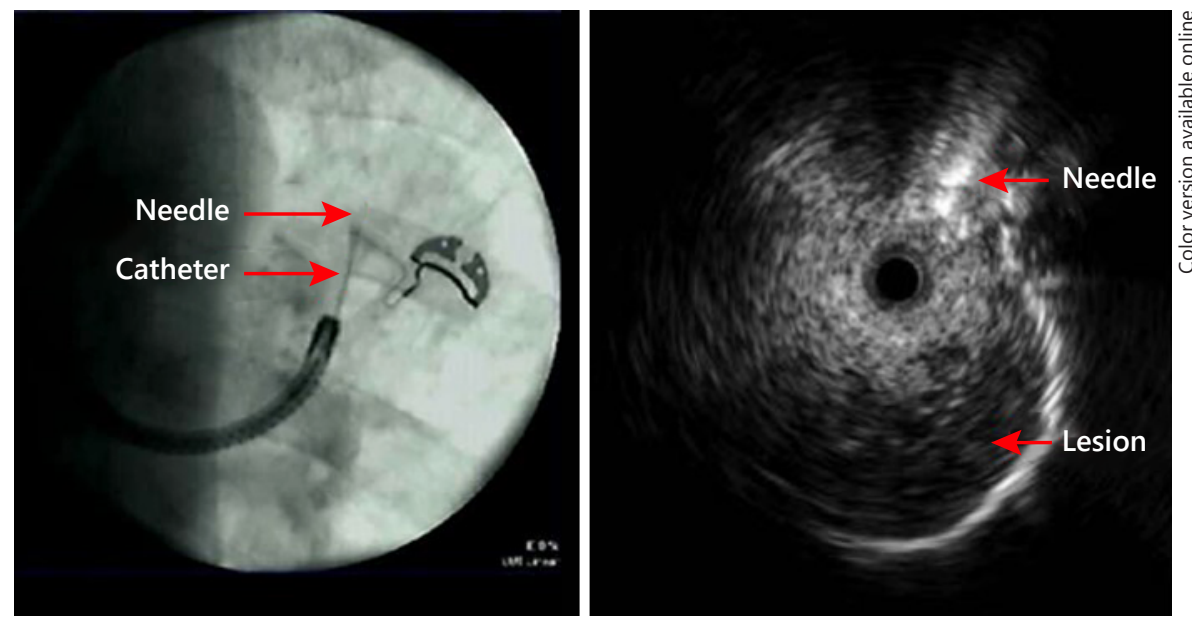

Table 1. Baseline demographics of 23 patients

\begin{tabular}{lc}
\hline Age, years & $68.0 \pm 10.9$ \\
Gender & \\
$\quad$ Female & 52.2 \\
Male & 47.8 \\
Physical exam & $78.29 \pm 19.87$ \\
$\quad$ Weight, kg & $171.2 \pm 9.4$ \\
Height, cm & $26.7 \pm 6.2$ \\
Body mass index & \\
Smoking history & 13.0 \\
Never & 26.1 \\
Current & 56.5 \\
Previous & 4.3 \\
Not disclosed & $30.6 \pm 25.4$ \\
Number of pack years & \\
\hline
\end{tabular}

Values are expressed as mean $\pm \mathrm{SD}$ or $\%$.

\section{Results}

Twenty-three participants were recruited and underwent bronchoscopy. Baseline demographics can be found in Table 1. The mean diameter of the peripheral lung lesions was 3.6 (range 1.7-5.7) cm. Targeted lesions were located and accessed in all lobes of the lung. Radiographic specifics and lobar location of the lesions are presented in Table 2.

\section{Primary End Point}

The RT-EBUS-TBNA device was successfully inserted into the bronchoscope and advanced to the target lesion in all lobes of the lung. All peripheral lung lesions were able to be visualized using RT-EBUS-TBNA (Fig. 3). Ad-
Table 2. Lesion characteristics in 23 patients

$\begin{array}{lr}\text { Location } & \\ \text { Right upper lobe } & 45.8 \% \\ \text { Right middle lobe } & 8.3 \% \\ \text { Right lower lobe } & 8.3 \% \\ \text { Left upper lobe } & 16.7 \% \\ \text { Left lower lobe } & 20.8 \% \\ \text { Density } & \\ \text { Solid } & 95.8 \% \\ \text { Ground-glass component } & 4.2 \% \\ \text { Mean diameter, cm }( \pm \text { SD) } & 3.6 \pm 1.3 \\ \text { Positive bronchus sign } & 95.8 \%\end{array}$

equate cytologic samples were obtained on $100 \%$ of these lesions under RT-EBUS-TBNA, with the needle visualized within the lesion in all cases. The average time of the RT-EBUS-TBNA procedure, defined as sheath insertion into the bronchoscope and subsequent removal, was 28.6 (range 6.0-56.0) $\mathrm{min}$ and a total procedure time (including linear EBUS, RT-EBUS-TBNA, and CR-EBUS) of 62.1 (range 41.0-114.0) $\mathrm{min}$.

Diagnostic yield results are presented in Table 3. RTEBUS-TBNA was diagnostic in 16/23 cases (70\%); 12 of these 16 were malignant and the remaining 4 were ultimately confirmed as benign after initial cytopathologic findings of inflammation and subsequent complete resolution on follow-up CT imaging. Overall, 20/23 cases were performed with both RT-EBUS-TBNA and CREBUS. All 7 nondiagnostic RT-EBUS-TBNA cases underwent additional standard of care procedures with CREBUS (Olympus UM-S20-17S); 2 of these 7 (29\%) were diagnostic of neoplasm (squamous-cell carcinoma and 
Table 3. Diagnostic yield of RT-EBUS-TBNA and CR-EBUS

\begin{tabular}{rll}
\hline Case No. & RT-EBUS-TBNA & CR-EBUS \\
\hline 1 & adenocarcinoma & adenocarcinoma \\
2 & adenocarcinoma & adenocarcinoma \\
3 & adenocarcinoma & adenocarcinoma \\
4 & adenocarcinoma & not performed \\
5 & adenocarcinoma & adenocarcinoma \\
6 & adenocarcinoma & adenocarcinoma \\
7 & squamous-cell carcinoma & squamous-cell carcinoma \\
8 & non-small-cell carcinoma & non-small-cell carcinoma \\
9 & non-small-cell carcinoma & non-small-cell carcinoma \\
10 & non-small-cell carcinoma & not performed \\
11 & epithelioid neoplasm & epithelioid neoplasm \\
12 & melanoma & not performed \\
13 & acute inflammation & acute inflammation \\
14 & acute inflammation & acute inflammation \\
15 & acute inflammation & acute inflammation \\
16 & acute inflammation & \\
17 & nondiagnostic & acute inflammation \\
18 & nondiagnostic & nondiagnostic \\
19 & nondiagnostic & nondiagnostic \\
20 & nondiagnostic & nondiagnostic \\
21 & nondiagnostic & nondiagnostic \\
22 & nondiagnostic & nondiagnostic \\
23 & nondiagnostic & typical carcinoid \\
\hline & & squamous-cell carcinoma \\
\hline
\end{tabular}

a Observed on cytology with CT follow-up confirming nodule resolution.

typical carcinoid). The remaining 13 CR-EBUS cases were performed for additional confirmatory tissue immediately after a diagnostic RT-EBUS case as per protocol.

The average number of samples taken with RT-EBUSTBNA was 3.7 (2.0-7.0). Additional standard of care techniques used to collect samples with CR-EBUS included forceps (61.5\%), brush (38.5\%), lavage (23.1\%), and needle biopsy (46.2\%). In patients undergoing additional sampling techniques with CR-EBUS, there were an average of 4 different techniques used and the procedure lasted an additional 10.7 (range 1.0-32.0) min.

\section{Secondary End Points}

There were 10 AEs documented in 7 individual procedures, and 9 of these were considered minor. All 7 procedures with AEs included RT-EBUS-TBNA, and 5 of them also involved CR-EBUS. None of the AEs was related to anesthesia, or directly attributed to the study device or CR-EBUS. There were 5 episodes of mild hemoptysis, 2 cases of mild sore throat, and 1 participant complained of a mild cough. All minor events were self-limiting and the average time to onset of an $\mathrm{AE}$ was 1 day (range 0-6.0 days). The single participant with a major AE initially presented for the procedure with evidence of a postobstructive process and a lavage during the index procedure grew Pseudomonas. This patient developed a Pseudomonas pneumonia 2 days after the procedure and apart from this, all of the AEs were mild. All participants had resolution of their symptoms, and there were no deaths associated with the procedure. No pneumothorax or moderate to severe bleeding was encountered.

\section{Discussion}

The diagnosis of peripheral pulmonary lesions can be achieved by invasive maneuvers such as surgical biopsy and CT-TTNA, but with significant morbidity in comparison to minimally invasive techniques [13-16]. Although conventional radial EBUS allows for real-time imaging of the nodule, the currently available platforms do not allow for nodule sampling under direct ultrasound visualization. As a result, diagnostic yields have remained stagnant given the technological barriers regarding guided access of eccentric lesions and the fact that CR-EBUS cannot provide anatomic laterality. The device described here offers a novel opportunity to overcome these limitations by its ability to provide needle repositioning under real-time ultrasound guidance. In addition, although TBNA has been shown to improve diagnostic yield and reduce complications in peripheral bronchoscopy, given these same issues, it remains underutilized and so a guided sampling approach may improve its useability [8].

In this study, we describe the first real-time visualization and sampling technique for the biopsy of peripheral pulmonary lesions in humans using a novel device. Prior attempts at real-time peripheral sampling were limited by the inability to obtain angular separation between the ultrasound and the needle [17]. This problem was overcome by designing a sheath that allows separation of the radial probe and needle at approximately $10^{\circ}$, thereby permitting direct visualization of the needle under ultrasound. The results of our pilot study, using a hybrid radial EBUS system with a needle adjacent to the catheter that allows for real-time biopsy of peripheral pulmonary lesions, demonstrate that this technique is feasible and has an acceptable safety profile in this early prototype. 
The device was able to be successfully advanced into all lobes of the lung, and the needle was visualized during tissue acquisition in all cases. In addition, because the needle is clearly visualized in a specific position in relation to the target lesion, for the first time, anatomic localization of a lesion and subsequent accurate repositioning have become feasible. In CR-EBUS, the $360^{\circ}$ ultrasound image does not provide anatomic landmarks to determine anterior/posterior or medial/lateral positioning. This limits the diagnostic capabilities of this tool, especially in lesions eccentric to the airway. When advancing the needle during RT-EBUS-TBNA, the location of the nodule in relation to the needle is clearly defined, affording the bronchoscopist the ability to easily retract the needle and reposition the catheter.

There are limitations to this study. This pilot study was observational, with the primary end point being to identify the feasibility of a prototype RT-EBUS-TBNA. The study was not powered for diagnostic yield end points and so reporting of yield is to be considered as purely observational. Although all lesions were able to be visualized and sampled using the RT-EBUS system, 12 participants required CR-EBUS due to inadequate sampling. The CR-EBUS technique utilized additional tools for sampling and was able to obtain adequate samples from $92.3 \%$ of those attempted; however, these participants had nearly double the number of passes to obtain tissue (6.3 vs. 3.7). The adequacy estimation by ROSE methodology during RT-EBUS-TBNA was likely underestimated in our cohort since the number of passes was limited to allow for appropriate time for additional tissue sampling by means of standard techniques if needed. Given that diagnostic yield is a critical component of the device's capabilities, future generations of this product will warrant further investigation, with larger prospective trials that investigate diagnostic yield concentrating on eccentric lesions which continue to prove challenging (given the anatomic challenges discussed earlier). Although all lobes were accessed, the prototype catheter was somewhat stiff which made positional advancements and precise maneuvering challenging, and the ultrasound signal of the needle was of variable quality at times. Although the initial results of this pilot study are promising, given that the RT-EBUS-TBNA device is disposable, cost will be an important factor that needs to be further defined before production is planned and the technique is adopted. Future generations of this product should focus on catheter maneuverability, needle visualization, and human factors to optimize the learning curve and diagnostic yield.

\section{Conclusions}

In this multicenter, prospective pilot study, we demonstrated that RT-EBUS-TBNA is feasible and has a similar side effect profile to standard CR-EBUS. The RTEBUS-TBNA device was able to directly visualize and sample pulmonary lesions under continuous ultrasound for the first time in humans. Future generations of this device may improve usability. Once achieved, adequately powered studies are needed to further validate these findings as well as to determine the true diagnostic yield of this novel technique.

\section{Acknowledgements}

L.Y. had full access to all data in the study and takes responsibility for the integrity of the data and the accuracy of the data analysis. L.Y. and A.C. contributed equally to the design, data analysis and interpretation, and primary drafting and final approval of the manuscript. C.M., G.S., H.L., D.F.-K., A.L., J.T., N.P., and N.T. contributed substantially to the study design, data analysis, and writing of the manuscript.

\section{Statement of Ethics}

Institutional review board approval was obtained from all institutions (IRB00101395) prior to the study start date. FDA approval under an IDE was obtained to perform this in-human clinical study. All patients provided informed consent.

\section{Disclosure Statement}

Drs. Yarmus, Silvestri, Lee, Feller-Kopman, Pastis, and Chen received educational grants and/or consulting fees from Boston Scientific.

\section{Funding Sources}

This project was funded by Boston Scientific. Research reported in this publication was supported by the National Heart, Lung, and Blood Institute of the National Institutes of Health (NIH) under award No. T32HL007534. The content is solely the responsibility of the authors and does not necessarily represent the official views of the NIH. 


\section{References}

1 Rivera MP, Mehta AC, Wahidi MM. Establishing the diagnosis of lung cancer: diagnosis and management of lung cancer, 3rd ed. American College of Chest Physicians evidence-based clinical practice guidelines. Chest. 2013;143:e142S-e65S.

2 Heerink WJ, de Bock GH, de Jonge GJ, Groen HJ, Vliegenthart R, Oudkerk M. Complication rates of CT-guided transthoracic lung biopsy: meta-analysis. Eur Radiol. 2017 Jan; 27(1):138-48.

3 Grossi W, Masullo G, Londero F, Morelli A. Small incisions, major complications: videoassisted thoracoscopic surgery management of intraoperative complications. J Vis Surg. 2018 Jan;4:12.

4 Eberhardt R, Anantham D, Ernst A, FellerKopman D, Herth F. Multimodality bronchoscopic diagnosis of peripheral lung lesions: a randomized controlled trial. Am J Respir Crit Care Med. 2007 Jul;176(1):36-41.

5 Wiener RS, Schwartz LM, Woloshin S, Welch HG. Population-based risk for complications after transthoracic needle lung biopsy of a pulmonary nodule: an analysis of discharge records. Ann Intern Med. 2011 Aug;155(3): 137-44.

6 Chen A, Chenna P, Loiselle A, Massoni J, Mayse M, Misselhorn D. Radial probe endobronchial ultrasound for peripheral pulmonary lesions. A 5-year institutional experience. Ann Am Thorac Soc. 2014 May;11(4): $578-82$.
7 Wang Memoli JS, Nietert PJ, Silvestri GA. Meta-analysis of guided bronchoscopy for the evaluation of the pulmonary nodule. Chest. 2012 Aug;142(2):385-93.

8 Ost DE, Ernst A, Lei X, Kovitz KL, Benzaquen S, Diaz-Mendoza J, et al.; AQuIRE Bronchoscopy Registry. Diagnostic Yield and Complications of Bronchoscopy for Peripheral Lung Lesions. Results of the AQuIRE Registry. Am J Respir Crit Care Med. 2016 Jan;193(1):6877.

9 Tanner NT, Yarmus L, Chen A, Wang Memoli J, Mehta HJ, Pastis NJ, et al. Standard Bronchoscopy with Fluoroscopy vs. Thin Bronchoscopy and Radial Endobronchial Ultrasound for Biopsy of Pulmonary Lesions: A Multicenter, Prospective, Randomized Trial. Chest. 2018 Nov; 154(5):1035-43.

10 Chen A, Pastis N, Furukawa B, Silvestri GA. The effect of respiratory motion on pulmonary nodule location during electromagnetic navigation bronchoscopy. Chest. 2015 May; 147(5):1275-81.

11 Loo FL, Halligan AM, Port JL, Hoda RS. The emerging technique of electromagnetic navigation bronchoscopy-guided fine-needle aspiration of peripheral lung lesions: promising results in 50 lesions. Cancer Cytopathol. 2014 Mar;122(3):191-9.
12 Zhang W, Chen S, Dong X, Lei P. Meta-analysis of the diagnostic yield and safety of electromagnetic navigation bronchoscopy for lung nodules. J Thorac Dis. 2015 May;7(5): 799-809.

13 Larscheid RC, Thorpe PE, Scott WJ. Percutaneous transthoracic needle aspiration biopsy: a comprehensive review of its current role in the diagnosis and treatment of lung tumors. Chest. 1998 Sep;114(3):704-9.

14 Loubeyre P, Copercini M, Dietrich PY. Percutaneous CT-guided multisampling core needle biopsy of thoracic lesions. AJR Am J Roentgenol. 2005 Nov;185(5):1294-8.

15 Loubeyre P, McKee TA, Copercini M, Rosset A, Dietrich PY. Diagnostic precision of image-guided multisampling core needle biopsy of suspected lymphomas in a primary care hospital. Br J Cancer. 2009 Jun;100(11):17716.

16 Schreiber G, McCrory DC. Performance characteristics of different modalities for diagnosis of suspected lung cancer: summary of published evidence. Chest. 2003 Jan;123(1 Suppl):115S-28S.

17 Shinagawa N, Yamada N, Asahina H, Kikuchi E, Oizumi S, Kurimoto N, et al. Transbronchial Biopsy for Peripheral Pulmonary Lesions Under Real-time Endobronchial Ultrasonographic Guidance. J Bronchology Interv Pulmonol. 2009 Oct;16(4):261-5. 